NASA Technical Memorandum 101397

AIAA-89-0236

\title{
Microgravity Research in NASA Ground-Based Facilities
}

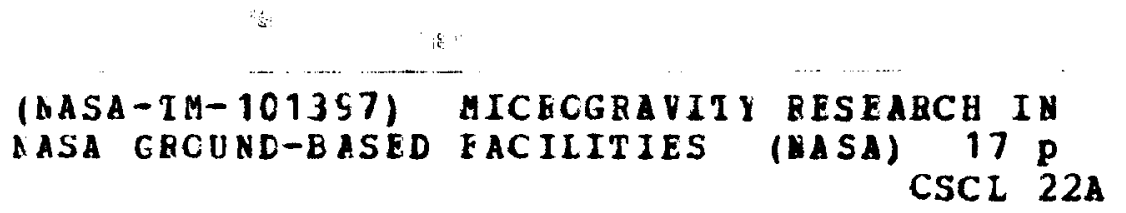

Jack Lekan

Lewis Research Center

Cleveland, Ohio

Prepared for the

27th Aerospace Sciences Meeting

sponsored by the American Institute of Aeronautics and Astronautics

Reno, Nevada, January .9-12, 1989 


\title{
MICROGRAVITY RESEARCH IN NASA GROUND-BASED FACILITIES
}

\author{
Jack Lekan \\ National Aeronautics and Space Adminstration \\ Lewis Research Center \\ Cleveland, Ohio
}

\begin{abstract}
An overview of reduced gravity research performed in NASA ground-based facilities sponsored by the Microgravity Science and Applications Program of the NASA Office of Space Science and Applications is presented. A brief description and summary of the operations and capabilities of each of these facilities along with an overview of the historical usage of them is included. The goals and program elements of the Microgravity Science and Applications Program are described and the specific programs that utilize the low gravity facilities are identified. Results from two particular investigations in combustion (flame spread over solid fuels) and fluid physics (gas-liquid flows at microgravity conditions) are presented.
\end{abstract}

\section{Introduction}

The Space Transportation System (Space Shuttle) and eventually the Space Station offer opportunities for long duration (hours, days, weeks) scientific research and technology development under microgravity conditions. The resources that are required to utilize such facilities demand thorough scientific justification and technological feasibility determinations before committing to a space-based experiment. Several NASA ground-based facilities are employed to obtain the baseline, normal gravity data and preliminary reduced gravity data (gravitational levels of $10^{-2} \mathrm{~g}$ to $10^{-6} \mathrm{~g}$ ) that are necessary to advance the scientific understanding and experiment concepts. The facilities that can provide the prerequisite low gravity environment include different drop towers and tubes and jet aircraft, each of which has its own capabilities to meet the needs of various types of research.

The recent two and one-half year period without Space Shuttle flights coupled with the potential longer range limitation on available Shuttle manifest opportunities for experiments increased the interest in expansion of the microgravity scientific data return from ground-based low gravity facilities. Thus, while space experiment hardware technology development and science requirements definition were continuing to be major activities in these facilities, many experiment programs were reexamined to determine how additional ground-based reduced gravity data might be utilized to enhance the success and value of limited space-based experiment time. Expanded groundbased test programs were defined and initiated for several experimental programs to obtain unique microgravity data which could be used to refine current analytical models of the processes and phenomena under investigation. These data and improved models may either stand-alone without the need for additional flight data or may be used to refine the final test matrices and experimental techniques that will be used in future space-based experiments.

This paper will discuss the NASA ground-based reduced gravity facilities and programs that support the Microgravity Science and Applications (MSA) Program of the NASA Office of Space Science and Applications. This discussion will include a summary of the background and capabilities of these facilities (which are located at the Lewis Research Center, the Lyndon B. Johnson Space Center, and the George C. Marshall Space Flight Center) and a brief description of the MSA Program. This paper will also address the types of 
research supported by the facilities and present results from two particular experiments in combustion and fluid physics.

\section{Background}

NASA initiated research in ground-based facilities to investigate the effects of reduced gravity on various processes and phenomena at the beginning of the space program in the late 1950's. These activities were prompted by the need of space-based system designers to overcome certain challenges that would be encountered by spacecraft in the low gravity conditions associated with orbital flight. Some of NASA's earliest studies were directed at space power and propulsion capabilities. In order to deal with the implementation of these systems which would include on-orbit fluid management and/or handling functions, it was critical to gain understanding of fluids under low gravity conditions. ${ }^{(1,2)}$ Besides technology development, other fundamental information such as spacecraft fire safety needed to be obtained. Investigations to gain the necessary knowledge/data were performed "on the earth" in drop towers, aircraft, and sounding rockets. Each of these facilities provided its own set of unique capabilities in terms of purity of gravity level (the level to which the effective gravity environment is reduced by providing a free fall or semi-free fall condition), duration of low gravity time, weight of experiment, researcher interaction, and the like. Man's understanding of many low gravity processes was greatly enhanced by the execution of thousands of test drops and hundreds of aircraft flights. While ground-based low gravity facilities continued to be utilized in the 1960's and early 1970's to advance research for new space transportation systems and advanced satellite applications, some of the actual low gravity research moved to the laboratory of space. While ground-based facilities offered an experiment time from 2 to 20 seconds, the Apollo program and Skylab offered much longer time duration for microgravity experimentation and these capabilites were utilized as required. ${ }^{(3)}$
Ground-based reduced gravity research was performed at a low level during the mid and late 1970's. This was due to the fact that the critical enabling low gravity technologies had been successfully developed and implemented and also some experimentation was also being performed in space. In fact during this time period, the Zero-Gravity Facility of the Lewis Research Center (LeRC) was shut down. However, in the 1980 's, over two decades after the birth of low gravity research, the United States Space Program entered the era of Space Shuttle Operations and Space Station planning. During this time, NASA's MSA Program was developed and has grown in stature to include seven research areas involving 135 researchers associated with over 60 public and private sector organizations. The activities in NASA ground-based facilities have parallelled the growth in the MSA Program. Research in these facilities have either reached or exceeded the levels of the pre-space era. Ground-based low gravity facilities now support the MSA Program in three different areas: (1) execution of ground-based science programs; (2) performance of precursor tests to define space experiment science requirements and conceptual designs; and (3) performance of tests for space experiment technology development and verification.

\section{lll. Ground-Based Facilities}

The NASA reduced gravity research facilities that support the MSA Program include two drop towers at LeRC, a drop tower and drop tube at the George C. Marshall Space Flight Center (MSFC), a KC-135 aircraft at the Lyndon B. Johnson Space Center (JSC), and a Learjet Model 25 at LeRC. Each of these facilities can provide a free fall or semi-free fall condition where the force of gravity is offset by linear acceleration, thus enabling a reduced gravity condition. Each of the facilities have different capabilities and characteristics that must be considered by an investigator in order to determine which facility is best suited for conducting a particular series of experiments. 


\section{Drop Towers and Drop Tubes}

Even though drop towers and tubes offer a relatively short term experiment time ranging from 2.2 to 5.18 seconds, this time span has been found to be sufficient for many phenomena in the disciplines of combustion, fluid dynamics and transport phenomena, and materials science. Also, since repeated experiments can be performed in these facilities, multistep phenomena that have longer characteristic times associated with them can sometimes be examined in a step-by-step process. Drop towers can accommodate large experimental packages with hardware weight up to several hundred kilograms. In all cases, the experiment is isolated from aerodynamic drag either through the use of a surrounding drag shield or by testing in an evacuated chamber. Drop tubes are enclosures that can accommodate small, uncontained material samples that are heated to the melt phase, and then can be resolidified while falling under microgravity conditions which are obtained by evacuating the enclosure. It is important to point out, that out of necessity, experiments in these facilities come to an abrupt halt at the end of the free-fall period. Deceleration levels can exceed $70 \mathrm{~g}$ 's.

The 2.2 Second Drop Tower at LeRC, as the name implies, provides 2.2 seconds of low gravity test time for experiment packages up to 125 kilograms of hardware weight by permitting the package to free fall a distance of 27 meters. A schematic diagram of this drop tower is shown in Figure 1. The facility consists of an eight floor building that is $\mathbf{3 0 . 5}$ meters tall. Contained within this building is the drop area, which is 27 meters tall with a cross section of 2.75 meters.

The experiment package, an example of which is shown in Figure 2, is enclosed in a drag shield which has a high rate of weight to frontal area and low drag coefficient. It aiso has three deceleration spikes attached to the bottom. The drag shield/experiment assembly is hoisted to the top of the building and suspended there by a highly stressed music wire which is attached to the release system. A drop is initiated by a system that notches the wire which in turn causes the wire to fail. The drag shield assembly is smoothly released and no measurable disturbances are imparted to the package by this procedure.

Accelerations of less than $10^{-5} \mathrm{~g}$ are obtained during the fall as the experiment package falls freely a distance of 20 centimeters within the drag shield. No guide wires or electrical cables are utilized in this procedure. Thus the only external force acting on the freely falling experiment package is the air drag associated with the relative motion of the package within the enclosure of the drag shield. Upon completion of the test, the drag shield is decelerated in a 2.2 meter deep sand pit by the deceleration spikes. The operating procedure of the 2.2 Second Drop Tower is such that 8 to 12 tests can be performed in one day.

Data from experiments in the 2.2 Second Drop Tower are acquired by high speed motion picture cameras with framing rates up to 1000 frames per second and onboard data acquisition systems which are utilized to record data supplied by instrumentation such as thermocouples, pressure transducers, and flow meters.

The 5.18 second Zero-Gravity Facility of LeRC, with its 132 meter free fall distance in a vacuum drop chamber represents a significant expansion in experiment sophistication and research capabilities when compared to the 2.2 Second Drop Tower. A schematic of this facility is shown in Figure 3. The ZeroGravity Facility houses a 6.1 meter diameter steel walled vacuum chamber that is 145 meters deep and provides the 132 meter drop distance required for 5.18 seconds of tree fall where accelerations of $10^{-6} \mathrm{~g}$ are attained.

Experiments with hardware weight of up to 450 kilograms are mounted in a one meter diameter drop bus as shown in Figure 4 . The drop bus is enclosed in 
a protective cover and suspended over the chamber by a single specially designed bolt in a hinged-plate release mechanism. A cover is placed over the chamber and an umbilical cable is attached to the top of the support shaft to maintain monitoring and control of the experiment systems prior to release. The entire chamber is then evacuated by a series of pumpodown procedures to a final pressure of $10^{-2}$ torr. Experiment entry into microgravity is initiated by shearing the bolt in the release mechanism. The bus falls free of drag in the near vacuum and is decelerated in a 6.1 meter deep container of small pellets of expanded polystyrene.

As in the Drop Tower, high speed motion picture cameras are again employed to acquire data. Also, other data such as pressures, temperatures, accelerations, etc. are either recorded on board with various data acquisition systems or are transmitted to a control room by a telemetry system that provides a capability of transmitting 18 channnels of continuous data. Due to the complexity of operations, one test is performed per day.

The 100-Meter Drop Tower at MSFC can simulate in-flight low gravity conditions for up to 4.27 seconds. A shematic of this facility is shown in Figure 5 . This drop tower has a total height of 101.7 meters and a freefall height of 89.5 meters.

Like the previously described drop towers, this facility is also designed to accommodate large experimental packages with sample weights of up to 180 kilograms. It utilizes the drag shield/experiment configuration as its mode of operation. Figure 6 is a schematic of this arrangement.

In the 100-Meter Drop Tower, the drag shield assembly is released and rides two guiderails downward until it enters a catch tube which is utilized for deceleration. This tube, which is $\mathbf{1 2 . 2}$ meters deep, decelerates the dragshield by the increase in air pressure which occurs by having the drag shield compress the air in the tube. Following this deceleration, the drag shield is stopped by horse-hair cushions in the bottom of the catch tube. To overcome the decelerating forces of air resistance and friction from the two guiderails which act on the drag shield, the drag shield is given a downward thrust by gas thrusters. Thus a properly balanced experiment package will float inside the drag shield. This system provides a g-level environment that can range from $4 \times 10^{-2} \mathrm{~g}$ to $10^{-5} \mathrm{~g}$ for a period of up to 4.2 seconds. Ten test drops can be performed in one day.

Test data is transmitted by telemetry to a control area for processing. High speed motion pictures cameras are also employed for data acquisiton, although a videotape system is also to be implemented.

The 100-Meter Drop Tube of MSFC provides up to 4.6 seconds of reduced gravity and is used extensively in the study of deep undercooling of metals and alloys. This tube is constructed of stainless steel and has an inner diameter of 0.254 meters. A schematic of this facility is shown in Figure 7. This drop tube has two devices for melting samples in the range of $\mathbf{5 0 0}$ to $\mathbf{3 5 0 0}$ degrees Centigrade. These are the electron beam furnace and the electromagnetic levitation furnace. Samples up to 5 millimeters in diameter and 800 milligrams in mass can be accommodated. The melting apparatus is located in a stainless steel bell jar which is located directly above the stainless steel drop tube. After the sample melts, it drops freely through the tube (actual fall is 105 meters) and is decelerated in a detachable fixture at the end of the fall.

Six turbomolecular pumps located equidistantly along the tube can provide a residual atmosphere within the tube of $8 \times 10^{-6}$ Torr. Thus accelerations as low as $10^{-6} \mathrm{~g}$ for a period of 4.6 seconds are possible. The tube can be backfilled with inert gas. Thermal history and the time of nucleation are acquired through 
infrared detectors and pyrometers. Closed-circuit television is utilized to record drop ejection and formation. A mass spectrometer analyzes the gas content of the tube and records the sample cooling rate.

\section{Alrcraft}

Specially modified jet aircraft flying parabolic (Keplerian) trajectories can provide significant increases in low-gravity experiment time when compared to drop towers and tubes but not without the penalty of higher gravity levels. For an experiment fixed to the body of an aircraft, accelerations in the range of $10^{-2} \mathrm{~g}$ can be obtained for up to twenty seconds. During one flight several trajectories are possible. While aircraft may not offer the microgravity levels for experimentation, they do offer the significant advantage of permitting researchers not only the opportunity to monitor their experiments in real-time, but also to reconfigure them between maneuvers. In larger aircraft such as the KC-135 at JSC, sufficient cabin volume allows certain experiments to be freefloated. This procedure generally produces accelerations below $10^{-3} \mathrm{~g}$ for a period of 5 to 15 seconds. These free-floated experiments are also isolated from the aircraft vibrations experienced by attached experiments.

The LeRC Learjet Model 25 is shown in Figure 8 along with its accompanying flight profile of a low gravity maneuver. This aircraft can be utilized for attached experiments only. Approximately 1.8 meters of cabin length are available for experiment mounting and researcher seating. Inherent lubrication limitations of this aircraft permit a maximum of 6 trajectories per flight. Intermediate acceleration levels ranging from $5 \times 10^{-2} \mathrm{~g}$ to $75 \times 10^{-2} \mathrm{~g}$ can also be attained by the Learjet.

The JSC KC-135A, a turbojet transport, has a much larger capacity than the Learjet both in terms of the number of trajectories and cabin space for experiments and researchers. Up to 40 parabolic trajectories can be performed in one flight. The research bay is $\mathbf{3 . 0 4}$ meters by $\mathbf{1 6 . 4}$ meters. This aircraft can also perform variable gravity level acceleration parabolas. Several experiments, including a combination of attached and free-floated hardware can be integrated for a single flight.

From the above summary of the description of the various NASA low gravity facilities it is evident that each facility has its own unique capabilities and limitations regarding the amount of test time, purity of reducedgravity levels, experiment size, operations, and researcher interaction. Thus one of the keys to effective utilization of each facility is the appropriate facility selection. Regardless of which facility is selected, each of them play a key role in support of the NASA MSA Program which will be briefly described in the next section.

\section{Microgavity Science and Appllcations Program}

NASA's MSA Program grew out of the former Materials Processing in Space Activity of the 1970's. This program has an overall goal to develop near earth space as a national resource. At the foundation of this goal is research into the effects of gravity on basic physical, chemical, and biological processes. The program is structured to address research in the following 7 major areas: Biotechnology, Combustion Science, Electronic Materials, Fluids and Transport Phenomena, Metals and Alloys, Glasses and Ceramics, and Polymers. An integrated team of NASA Centers, Universities, Industry, and Centers of Excellence are supported in the investigations of these disciplines.

Research is being pursued in these areas since the effect of gravity pervades virtually every physical science. It is important to understand the effects of gravity on convection, buoyancy, and sedimentation. 
Experiments of fluid physics and combustion processes under weightlessness can help reveal subtle interactions among processes that are not observable under normal gravity. Gravity also affects all aspects of materials processing from the liquid or gaseous state. For example, gravity-driven fluid flows redistribute dopant or alloy additions which limit crystalline perfection and device efficiency. Also, accurate measurements of many thermophysical properties such as diffusion coefficients or viscosity and heat capacity near the critical point are precluded due to the gravity-induced effects such as hydrostatic pressure which compress or collapse fluid specimens and buoyancy-driven flows which induce uncontrolled/ undersired mixing.

Research in the MSA Program includes the program elements of both Ground-Based Research and Science Flight Experiments and also the areas of Advanced Technology Development and Space Station Facility Development. Ground-based low gravity facilities play a vital role in the support of these program elements. The following sections will identify those programs that utilize these facilities and will present results from two particular investigations.

\section{Programs Supported By NASA Ground-Based Facilities}

The cost of spaced-based experiments coupled with limited opportunities for actual time in space has created a situation where every effort be made to maximize the value of the data that can be obtained in space. Thus the processes and difficulties that are likely to be encountered in space experiments must be as fully understood and prepared for prior to actual flight. Over the last several years, NASA ground-based facilities have been utilized to provide more extensive low gravity data to aid in this understanding. Groundbased research has been instrumental in the refinement of technologies, hardware, and experiment test matrices. The following will identify a number of experiments that are executed in the NASA facilities in support of the MSA Program. It would not be practical to list and discuss all the experiments.

The type of reduced gravity research performed at a NASA center is based on the particular expertise of the center plus the capabilities of the specitic facilities that are available. At LeRC, the facilities play a very effective role in low gravity combustion and fluids research. The 2.2 Second Drop Tower offers rapid experiment turn-around time, low cost of operation and significant researcher involvement. Thus it is an excellent facility to conduct experiments that are exploratory or ill-defined or do not require more than 2.2 seconds of test time. Well-defined experiments that require more than 2.2 seconds of test time and highpurity low gravity levels are suitable candidates for the Zero-Gravity Facility. This facility also provides a high level of experiment sophistication. Experiments which require longer test times and can also tolerate less than minimum low gravity levels and/or require operator interaction are considered for testing on the Learjet. The distribution of some of the experiments among the LeRC reduced gravity facilities is shown below, where D represents the Drop Tower, $Z$, the Zero-Gravity Facility, and L, the Learjet:

Premixed Gas Combustion - D

Diffusion Flame Combustion - D,Z

Fuel Droplet Vaporization - D

Pool Fires Combustion - D,Z

Droplet Combustion - D,Z

Solid Fuel Flame Spread - D,Z

Particle Cloud Combustion - D,L

Pool Boiling - Z

Surface Tension Driven Convection - $D, L, Z$

Two-Phase Flow - D,L

The 100 Meter Drop Tube and Drop Tower at MSFC have been used effectively in the execution of reduced gravity materials science experimentation. The drop tube is used extensively for convection 
research in which extremely small samples are studied. This facility can provide deep undercooling for containerless processing experiments that require materials to remain in a liquid phase when cooled below the normal solidification temperature. Table 1 contains a list of metals that have been examined in a series of undercooling experiments. ${ }^{(4)}$ While experimentation in this facility also includes high temperature intermetallics and stainless steels, the major emphasis has been on undercooling and solidification phenomena in the Nb-based systems where several metastable structures have been observed. ${ }^{(5)}$ Research in the 100 Meter Drop Tower has predominantly adressed immiscible metals.

The JSC KC-135 aircraft is an extensively utilized research laboratory that supports several disciplines in the MSA Program. A range of $g$ levels can be provided as experiments can be fixed to the body of the aircraft or free-floated in the cabin. The aircraft can accommodate large experiments. It provides a large number of low gravity maneuvers (up to 40 ) per flight. Also, as previously stated, investigators can witness, operate, and reconfigure their experiment during the course of a flight. An example of the type of experiments that benefit from this capability provided by an aircraft laboratory is in materials science where directional solidification furnaces and containerless processing systems are utilized. The following is a small sample of KC-135 reduced gravity test programs:

Separation of Aqueous Two-Phase Polymer Systems

Directional Solidification of Flake and Modular Cast iron

Bubble Motion in a Rotating Liquid

Superalloy Microstructural Variations Induced by Gravity Level During Directional Solidification

Gas Jet Diffusion Flames

Critical Point Wetting of Immiscible Fluids

Three-Axis Acoustic Levitator Tests

Microgravity Crystallization of Macromolecules
The experiments/programs identified above were intended to show the range and types of research that are supported by the NASA reduced gravity facilities. Because of the large number of programs that utilize the facilities, it would be impossible to provide detailed experimental descriptions in this paper. However, for purposes of illustrating the types of research results that can be accomplished, a brief description of two of the projects that utilize the facilities at LeRC are dicussed in the next sections. One of the experiments is in combustion and the other is in fluids. It will be shown that one of the keys of the success of these experiments is their effective utilization of the facilities. In these two examples, the needs of the experiments were clearly matched to the facility capabilities.

Combustlon. Flame spread over solid fuels has been examined extensively in the drop towers at LeRC. Flame spread and extinction in low gravity are investigated to improve fundamental understanding of the combustion of solid materials and to predict materials flammability in low gravity environments. Flame spreading and flame extinction involve a complex interaction of chemical processes which includes the flame and the decomposition of the solid fuel and several modes of thermal transport. In normal gravity combustion, the flow field in the vicinity of the flame is dominated by buoyancy-induced flow due to high temperature gradients. In reduced gravity conditions, the buoyant flows are reduced and flame spread rates and flame extinction limits are different. Under these conditions, small momentum flows associated with the evaporation and expansion of the conducted fuel can be observed. In order to obtain an environment where the buoyant gas-phase motion can be controlled in a flame spreading experiment, a high purity reduced gravity level is required. This environment can be obtained in the LeRC Zero-Gravity Facility and the 2.2 Second Drop Tower.

Recent efforts in this area of research have focused on flame spreading over a thermally-thin 
cellulose fuel in microgravity under quiescent flow conditions and slow forced-flow conditions in the LeRC 2.2 Second Drop Tower and Zero-Gravity Facility respectively. ${ }^{(6)}$ In both cases the fuel was ignited and burned under a variety of oxygen/nitrogen atmospheres at one atmospheric pressure. Results of this experimental work in microgravity and also in normal gravity together with existing data at elevated gravity levels and in high velocity forced flow were utilized to examine extinction and flame spread behavior over the entire range of characteristic relative velocities between the flame and the oxygencontaining atmosphere.

A key result from this study is shown in Figure 9. This figure represents a flammability map using oxygen percentage and characteristic relative velocities as coordinates. The slow velocity data points are obtained from microgravity experiments, while one data point is the normal gravity natural convection blowoff limit. The remaining data points in the higher velocity blowoff branch are from forced convective experiments in wind tunnels. The extinction boundary in the flammability map consists of a high velocity branch and a low velocity branch. Blowoff extinction is a relatively well understood phenomena that is associated with the cooling of the flame by convective flow and the relatively short residence time of oxidant compared to the time for the chemical reaction to occur. The extinction depicted in the left side of the figure which results from decreasing the opposed flow relative velocity, however, is a new and different phenomena. This extinction mode has been labeled as "quenching". As the characteristic relative velocity decreases, the flame standoff distance increases which then diminishes the heat feedback from the flame and the burning rate. The rate of heat loss from the flame and fuel surface becomes significant and the flame temperature decreases and eventually there is extinction.

The implications of this work to applications stem from the fact that the fundamental low oxygen limit (approximately $15 \%$ ), or most flammable flow condition, occurs within the characteristic relative velocity range between 6 to $10 \mathrm{~cm} / \mathrm{sec}$. This flow condition is equivalent to a gentle draft in microgravity and can easily be found inside a habitated spacecraft environment.

Fluld Dynamics. One of the areas of study in fluid dynamics is multiphase flow. Multiphase flows involve the simultaneous forced flow of more than one phase of a single fluid or multiple fluids through a conduit. These processes are often accompanied by heat and mass transfer between phases which can influence flow behavior. The types of flow patterns and the transitions between the flow patterns have been the topic of many studies because of their importance in engineering liquid transfer systems. Some of the mechanisms of transition are reasonably well understood for flow pipes on earth, where it has been shown that body forces largely control the behavior observed. Under microgravity conditions these body forces are suppressed and the basic flow pattern and transitions are not currently understood or predictable. In order to develop a physical model for these processes, experimental data must be obtained regarding flow regime characteristics (distribution of liquid and gas phases in a flow channel), pressure drops and fluctuations, heat transfer, and other parameters. There are several direct space-based applications for obtaining low gravity data for multiphase flow including cryogenic transter and storage, heat transfer associated with space nuclear power facilities and the design and operation of a thermal bus for the space station.

Experimental hardware for reduced gravity research in this area can be large in size. Also a relatively "long" amount of experimental time is required for examination of multiphase phenomena under reduced gravity conditions. Finally, the required purity of the gravity levels for this type of fluids research has been found to not be as low as that necessary for other research such as the combustion work described 
earlier. Thus, an aircraft becomes an ideal testbed for investigations of this nature.

The LeRC Learjet has been utilized in the investigation of a two-phase flow system under low gravity conditions. (7) For initial work in this area, air and water were selected as fluids to investigate adiabatic flow conditions. These fluids were selected due to simplicity in terms of considerations such as toxicity. Experimental data obtained during a series of tests included flow rates, temperatures, time dependent pressure drops, and high speed movie films. This experiment provided data on flow patterns, velocities of slugs, bubbles and interfacial waves, and sizes of bubbles and slugs.

Examples of the photographic data from this investigation are shown in Figures 10 and 11. Figure 10 shows photographs of single frames taken from high speed films (400 frames/second) where tests were made under both normal and low gravity, low flow rate conditions: gas velocity of $0.16 \mathrm{~m} / \mathrm{s}$ and liquid velocity of $0.08 \mathrm{~m} / \mathrm{s}$. Flow is from left to right. In normal gravity, the gas and water phases exist as layers with gas on top of the water due to the strong force of buoyancy. The reduced gravity condition, however, is dominated by surface tension which pulls the liquid around the tube perimeter and separates the gas into pockets. These well-established stable spherically nosed bubbles which are axisymmetric, move along the pipe separated by clear liquid slugs and, in some cases, the liquid slugs contain dispersed smaller gas bubbles. As gas flow rates are increased, bubbles become longer until a condition appears that is very close to transition between slug and annular flow. As annular flow is reached, long stretches of nearly smooth film are occasionally disrupted by a slow moving high amplitude wave. Eventually at higher gas rates, the film is very wavy with occasional large roll waves sweeping by at velocities which approach the gas velocity.

Conditions which exist at higher liquid flow rates where liquid velocities approach $1 \mathrm{~m} / \mathrm{s}$ can be seen in the example of Figure 11. Gas is dispersed in the water as bubbles. Again in normal gravity, buoyancy dominates and forces bubbles to the top. In reduced gravity, the bubbles are dispersed throughout the cross-sectional area of the tube. As the gas flow rate is increased, the bubbles become smaller and more closely packed but are still dispersed. With increasing gas flow rates, the condition of high flow rate slug flow is achieved where a moving highly aerated liquid slug is separated from the next slug by a thick, wavy liquid film which carries the bubbles.

Visual observations such as those described above are very useful in formulating simple models to predict the transitions from one regime to another and also for defining flow regimes in reduced gravity. Eventually this study will be extended to include effects of heat transfer as additional series of tests will be conducted on the Learjet.

\section{Vl. Concluding Remarks}

The NASA ground-based reduced gravity facilities have played a vital role in advancing the understanding of low gravity processes and phenomena, have enabled the definition and development of spacebased technology, and have enhanced the understanding of basic biological, chemical, and physical processes. Low gravity research on the earth will continue to grow in importance through the Space Shuttle and Space Station era by providing critical, economical pre-flight scientific and technological support.

\section{References}

1. Stark, J.A.: Low-G Fluid Behavior Technology Summaries. NASA CR-13746, December 1974.

2. Aydelott, J.C. and Symons, E.P.: LeRC Reduced Gravity Fluid Management Technology Program. 1980 JANNAF Propulsion Meeting, 
vol. 5, CPIA-PUBL-315-VOL-5, K.L. Strange, ed. 1980, pp. 21-34.

3. Kimzey, J.H.: Skylab Experiment M479, Zero Gravity Flammability. Proceedings of the Third Space Processing Symposium on Skylab Results, Vol. 1, NASA TM X-70252, 1974, pp. 115-130.

4. Hofmeister, W.H., Robinson, M.B., and Bayuzick, R.J.: Undercooling of Pure Metals in a Containerless, Microgravity Environment. Appl. Phys. Lett., vol. 49, no. 20, Nov., 1986, pps. 1342-1344.
5. Hofmeister, W.H., Evans, N.D., Bayuzick, R.J., and Robinson, M.B.: Microstructures of NiobiumGermanium Alloys Processed in Inert Gas in the 100 Meter Drop Tube. Metallurgical Transaction A, vol. 17A, Aug. 1986, pp. 1421-1428.

6. Olson, S.L., Ferkul, P.V. and T'ien, J.S.: NearLimit Flame Spread over a Thin Solid Fuel in Microgravity. Twenty-Second Symposium (International) on Combustion, Seattle WA, 1988.

7. Dukler, A.E., Fabre, J.A., McQuillen, J.B., and Vernon, R.: Gas-Liquid Flow at Microgravity Conditions: Flow Patterns and Their Transitions. Int. J. Muttiphase Flow, vol. 14, no. 4, 1988, pp. $389-400$.

Table 1. Materials utilized in 100-Meter Drop Tube undercooling experiments-materials tested with source, nominal purity, mass size, cooling time, and estimated undercooling.

\begin{tabular}{|c|c|c|c|c|c|c|c|}
\hline Metal & Source & $\begin{array}{l}\text { Purity } \\
\text { (\%) }\end{array}$ & $\begin{array}{l}\text { Mass } \\
(\mathrm{g})\end{array}$ & $\begin{array}{c}\text { Drop } \\
\text { diameter } \\
(\mathrm{mm})\end{array}$ & $\begin{array}{l}\text { A verage time to } \\
\text { recalescence } \\
\text { (s) }\end{array}$ & $\begin{array}{l}\text { Undercouling } \\
\text { (K) }\end{array}$ & $\% T_{m}$ \\
\hline$T_{i}$ & Johnson \& Matthey & 99.98 & 0.180 & 1.8 & 4.5 & 350 & 18 \\
\hline $\mathbf{Z r}_{\mathbf{r}}$ & Johnson \& Matthey & 99.8 & 0.390 & 5.0 & 3.5 & 430 & 20 \\
\hline $\mathbf{N b}$ & Materials Research Corp. & 99.99 & $0.360-0.720$ & $4.4-5.6$ & $2.1-2.8$ & 480 & 17.5 \\
\hline Mo & GTE & 99.9 & 0.575 & 4.9 & 2.6 & 520 & 18 \\
\hline Ru & Englehard & 99.8 & $0.73-0.88$ & $4.8-5.1$ & $1.5-3.8$ & $100-330$ & $4-13$ \\
\hline $\mathbf{R h}$ & Englehard & 99.99 & 0.067 & 2.3 & 3.2 & 450 & 20 \\
\hline $\mathbf{H f}$ & Teledyne Wah Chang Albany & $\stackrel{97}{(3 \% \mathrm{Zr})}$ & 0.600 & 4.6 & 2.2 & 450 & 18 \\
\hline Ta & Materials Research Corp. & 99.99 & 0.400 & 3.7 & 1.3 & 650 & 20 \\
\hline Ir & Englehard & 99.7 & 0.430 & 3.3 & $1.4-2.1$ & $270-340$ & $10-12.5$ \\
\hline $\mathbf{P t}$ & Englehard & 99.99 & 0.175 & 2.6 & 3.0 & 380 & 18.5 \\
\hline
\end{tabular}




\section{ORIGINAL PAGE IS \\ OF POOR QUALITY}
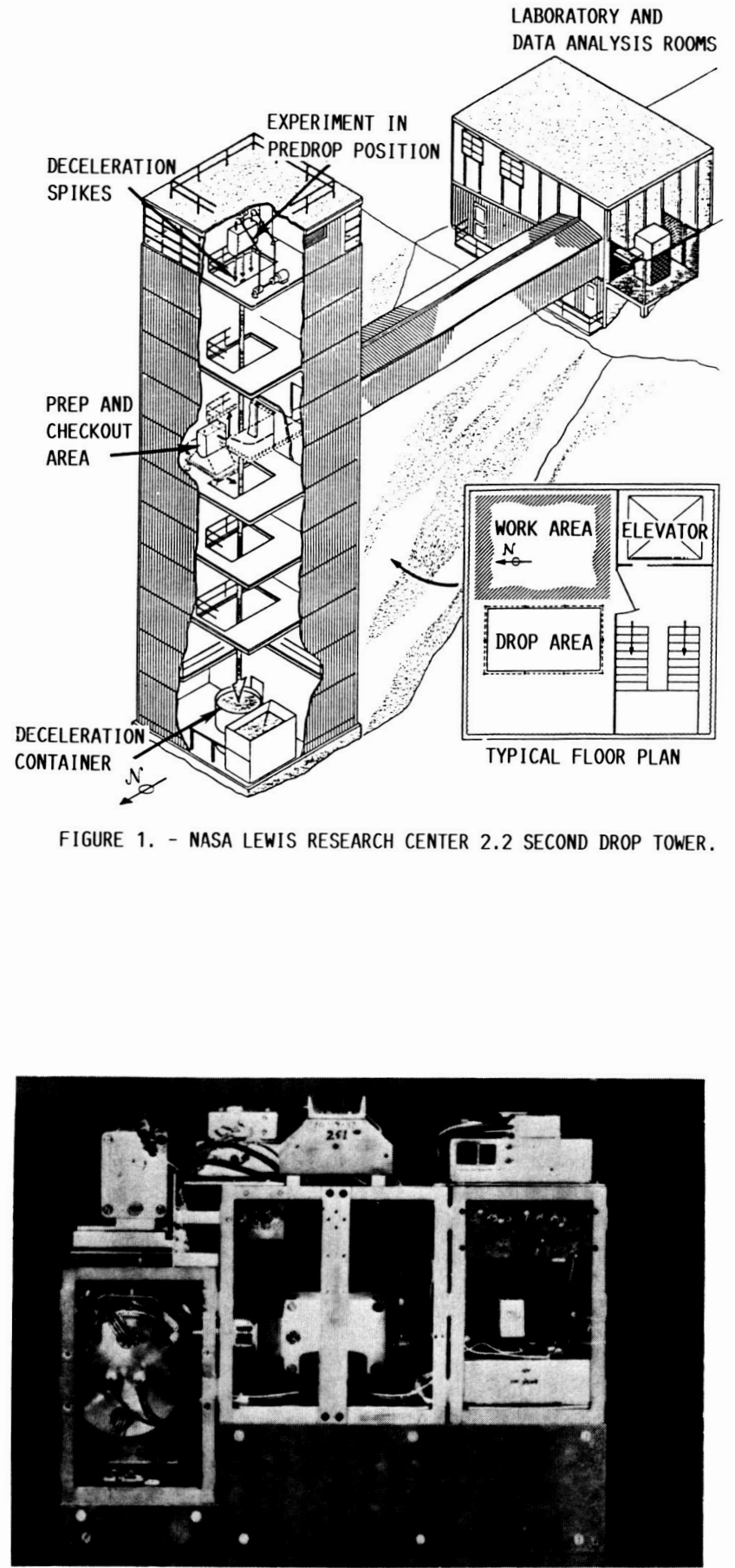

FIGURE 2. - EXPERIMENT PACKAGE FOR THE 2.2 SECOND DROP TOWER DROPLET COMBUSTION EXPERIMENT. 


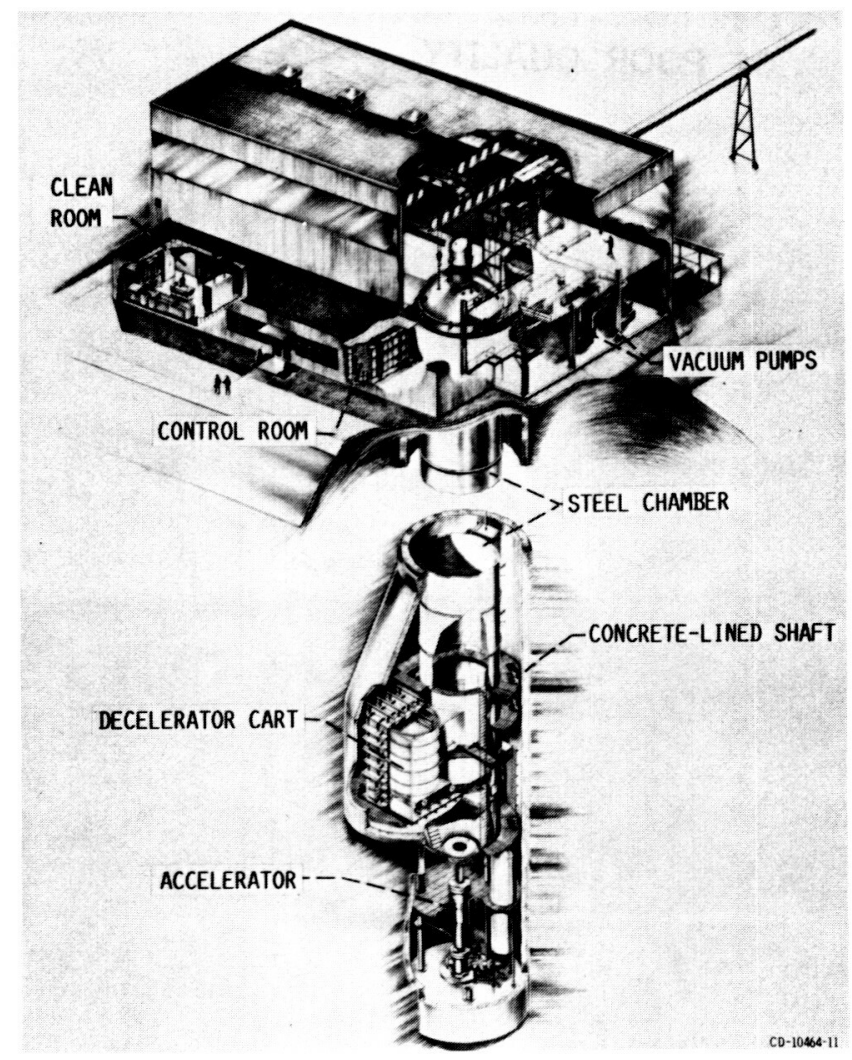

FIGURE 3. - CUTAWAY OF NASA LEWIS RESEARCH CENTER ZERO GRAVITY FACILITY.

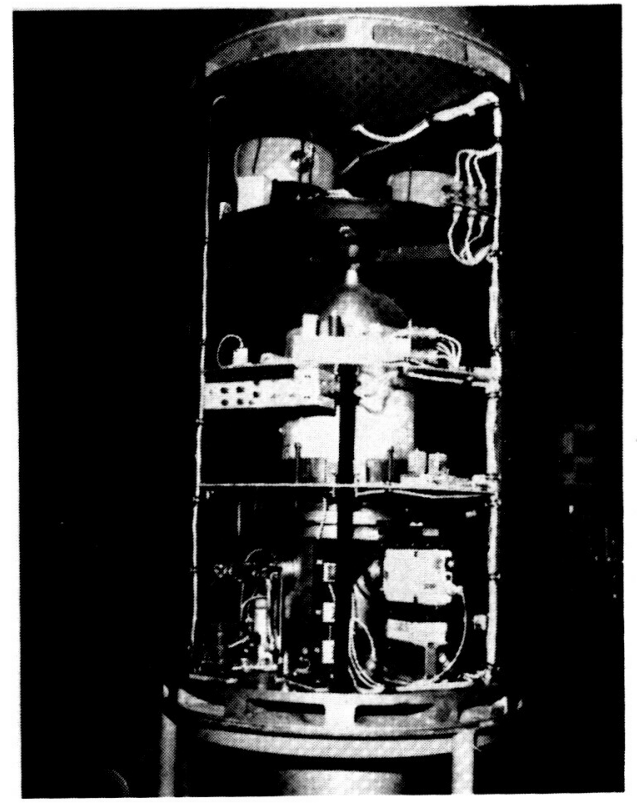

FIGURE 4. - EXPERIMENT VEHICLE FOR THE ZERO-GRAVITY FAC II ITY-COMBUSTION CHAMBER. 


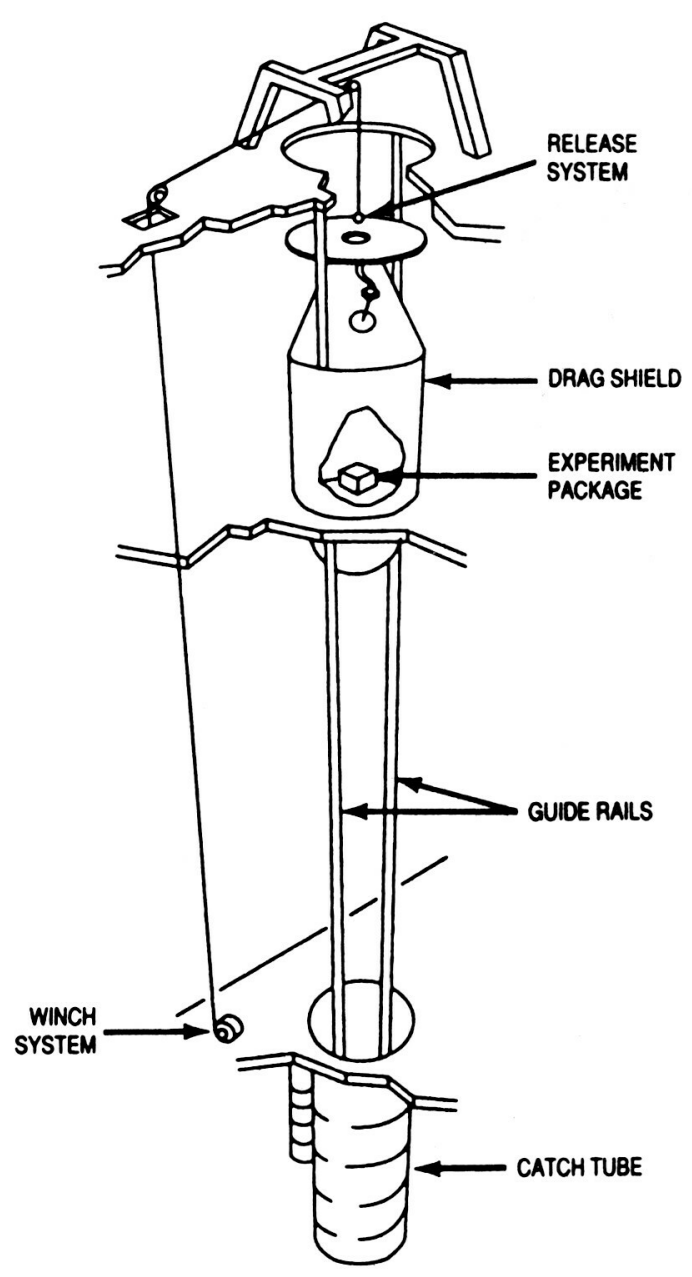

FIGURE 5. - MARSHALL SPACE FLIGHT CENTER 100-METER DROP TOWER.

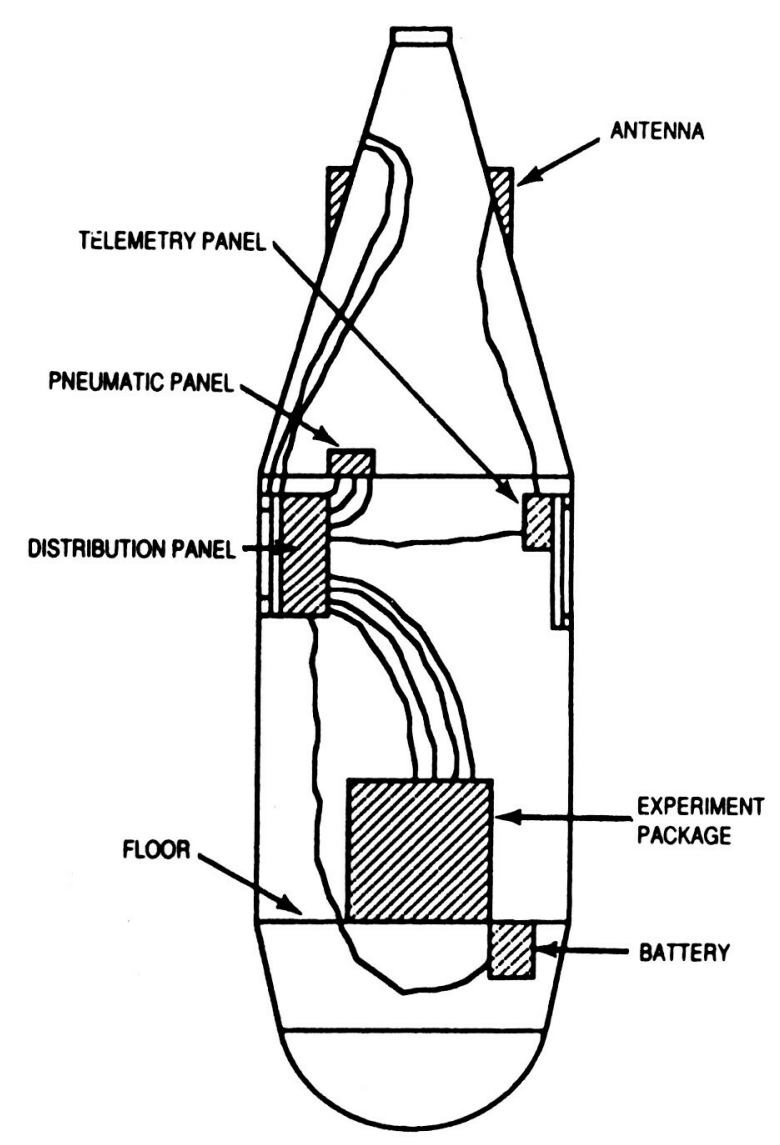

FIGURE 6. - DRAG SHIELD/EXPERIMENT CONFIGURATION FOR THE 100-METER DROP TOWER. 


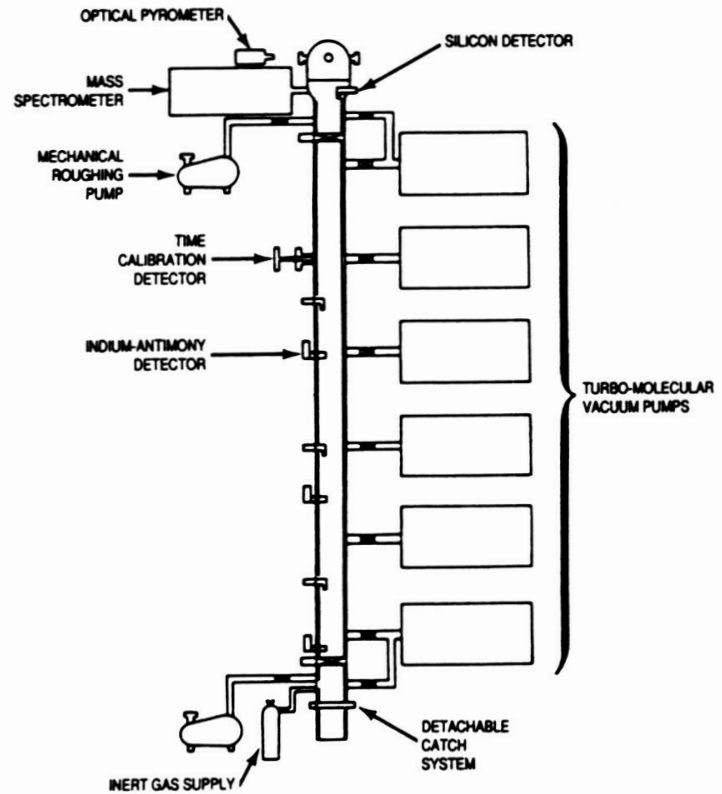

FIGURE 7. - MARSHALL SPACE FLIGHT CENTER 100-METER DROP TUBE.

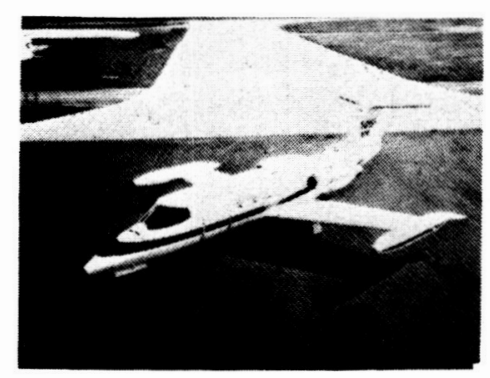

LERC MODEL 25 LEARJET

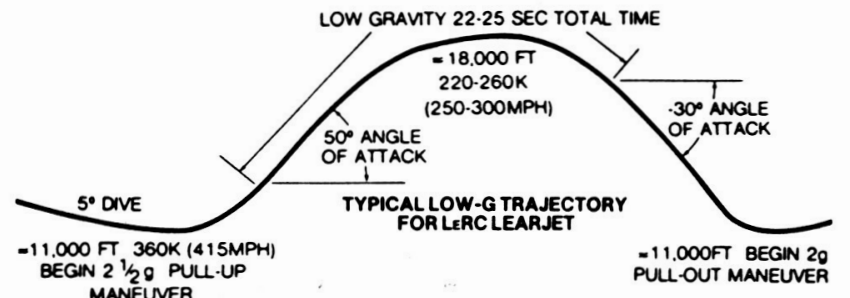

FIGURE 8. - NASA LEWIS RESEARCH CENTER LEARJET RESEARCH LABORATORY.

MICROGRAVITY DATA

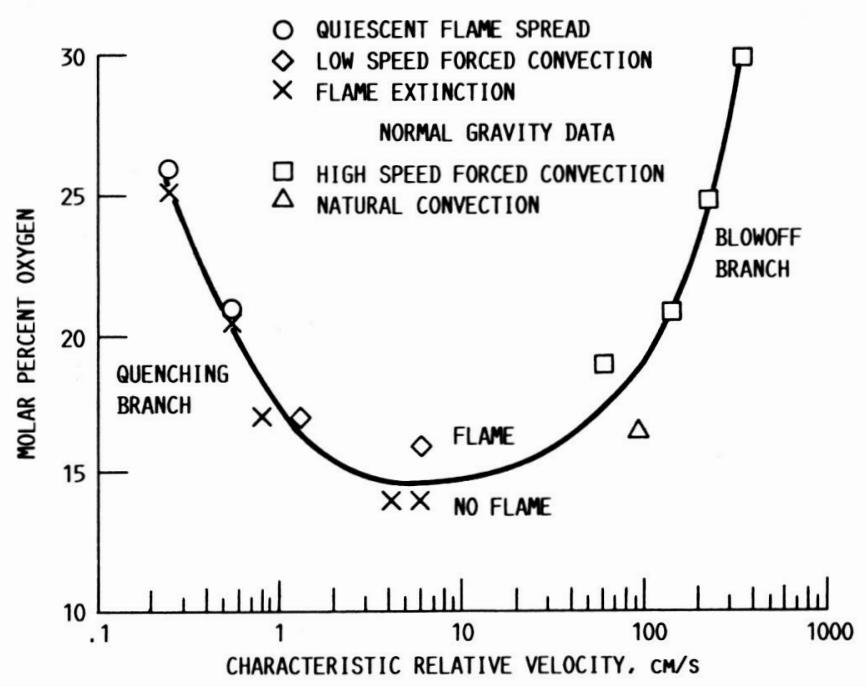

FIGURE 9. - EXTINCTION BOUNDARY FOR FLAME SPREAD OVER A THIN CELLULOSE FUEL. 


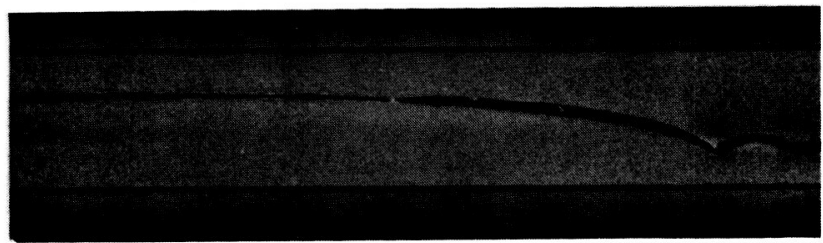

NORMAL GRAVITY

STRATIFIED

SMOOTH FLOW

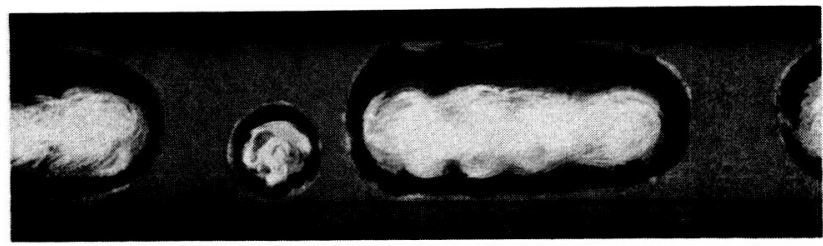

REDUCED GRAVITY

SLUG FLOW

FLUIDS: AIR AND WATER

TUBE DIAMETER: $1.27 \mathrm{cM}$

SUPERFICIAL GAS VELOCITY: $0.16 \mathrm{~m} / \mathrm{s}$

SUPERFICIAL LIQUID VELOCITY: $0.08 \mathrm{~m} / \mathrm{S}$

FIGURE 10. - LOW FLOW RATE EXAMPLE OF TWO-PHASE SYSTEM.

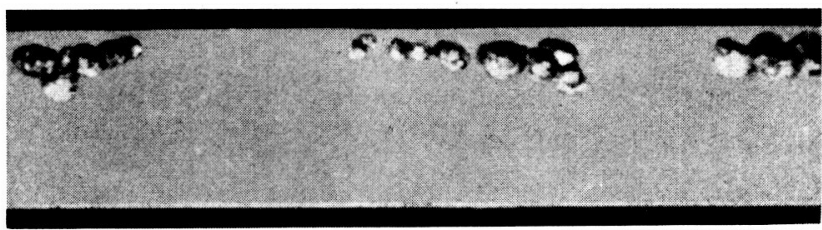

NORMAL GRAVITY

BUBBLY FLOW

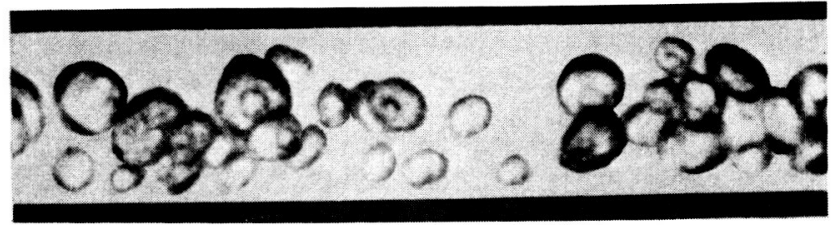

REDUCED GRAVITY

BUBBLY FLOW

FLUIDS: AIR AND WATER

TUBE DIAMETER: $1.27 \mathrm{cM}$

SUPERFICIAL GAS VELOCITY: $0.16 \mathrm{~m} / \mathrm{s}$

SUPERFICIAL LIQUID VELOCITY: $0.90 \mathrm{~m} / \mathrm{s}$

FIGURE 11. - HIGH LIQUID FLOW RATE EXAMPLE OF TWO-PHASE SYSTEM. 


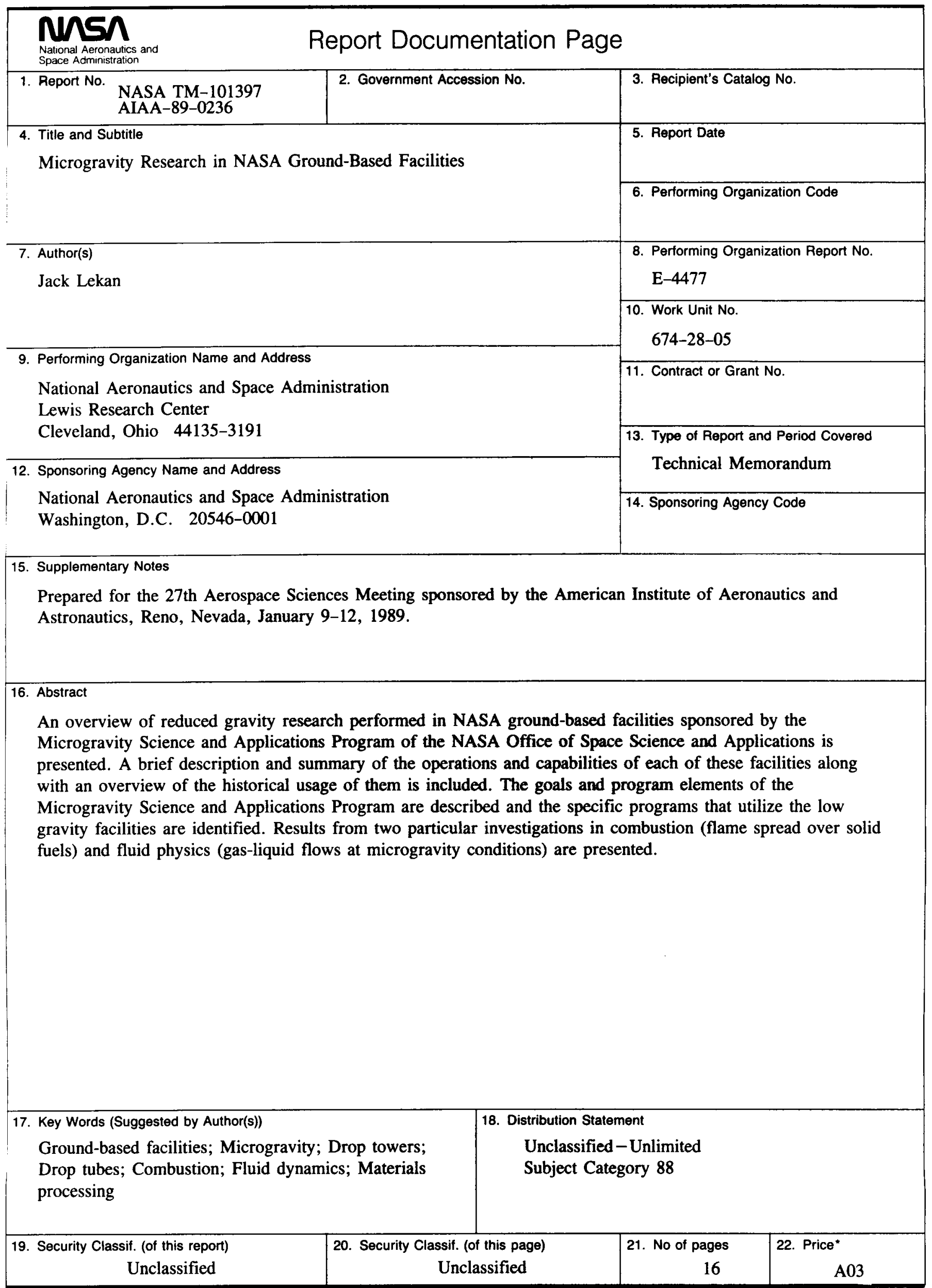

